\title{
Note on the problem of Ramanujan's radial limits
}

\author{
Bin Chen ${ }^{1,2}$ and Haigang Zhou ${ }^{1 *}$
}

"Correspondence:

haigangz@tongji.edu.cn

'Department of Mathematics,

Tongji University, No. 1239, Siping

Road, Shanghai, 200092, China

Full list of author information is

available at the end of the article

\begin{abstract}
Ramanujan in his deathbed letter to GH Hardy concerned the asymptotic properties of modular forms and mock theta functions. For the mock theta function $f(q)$, he claimed that as $q$ approaches an even order $2 k$ root of unity $\zeta$,$$
\lim _{q \rightarrow \zeta}\left(f(q)-(-1)^{k}(1-q)\left(1-q^{3}\right)\left(1-q^{5}\right) \cdots\left(1-2 q+2 q^{4}-\cdots\right)\right)=O(1)
$$

where $(1-q)\left(1-q^{3}\right)\left(1-q^{5}\right) \cdots\left(1-2 q+2 q^{4}-\cdots\right)=\prod_{n=1}^{\infty} \frac{1-q^{n}}{\left(1+q^{n}\right)^{2}}$. Recently, Folsom, Ono and Rhoades have proved two closed formulas for the implied constant and formulated an open problem which is related to their two theorems. In this note, we give a new proof on the problem of the two theorems by using some results about the generating functions of convex compositions given by GE Andrews and Appell-Lerch sums.
\end{abstract}

MSC: 11F37; 11F03; $11 \mathrm{Fg9}$

Keywords: modular forms; mock theta functions; generating functions

\section{Introduction}

In his deathbed letter to Hardy, Ramanujan gave no definition of mock theta functions but just listed 17 examples and a qualitative description of the key properties that he had noticed. Since that time, many papers studying the 17 specific examples have been written by many famous mathematicians such as Watson, Selberg and Andrews [1]. Due to the work of Zweger [2, 3], Bringmann and Ono [4, 5], Zagier [6] and others, we are able to recognize Ramanujan's mock theta functions as holomorphic parts of certain harmonic weak Maass forms of weight $1 / 2$, originally defined by Bruinier and Funke [7]. This realization has resulted in many applications in combinatorics, number theory, physics and representation theory.

While the theory of the weak Maass forms has led to a flood of applications in many disparate areas of mathematics, it is still not the case that we fully understand the deeper framework surrounding the contents of Ramanujan's last letter to Hardy. Here we revisit Ramanujan's original claims from his deathbed letter [8], which begins by summarizing the asymptotic properties, near roots of unity, of the Eulerian series which were modular forms. He then asked whether others with similar asymptotic were necessary for the summation of a modular form and a function which is $O(1)$ at all roots of unity. In fact, the

(O2014 Chen and Zhou; licensee Springer. This is an Open Access article distributed under the terms of the Creative Commons Attribution License (http://creativecommons.org/licenses/by/2.0), which permits unrestricted use, distribution, and reproduction in any medium, provided the original work is properly cited. 
recent work by Griffin et al. [9] has confirmed that there are no weakly holomorphic modular forms which exactly cut out the singularities of Ramanujan's mock theta functions.

Claim (Ramanujan [8]) As q approaches a primitive even order $2 k$ root of unity $\zeta$ radially within the unit disk, then

$$
\lim _{q \rightarrow \zeta}\left(f(q)-(-1)^{k}(1-q)\left(1-q^{3}\right)\left(1-q^{5}\right) \cdots\left(1-2 q+2 q^{4}-\cdots\right)\right)=O(1),
$$

where the mock theta function $f(q)$ is defined by

$$
f(q):=1+\sum_{n=1}^{\infty} \frac{q^{n^{2}}}{(1+q)^{2}\left(1+q^{2}\right)^{2} \cdots\left(1+q^{n}\right)^{2}},
$$

and

$$
\prod_{n=1}^{\infty} \frac{1-q^{n}}{\left(1+q^{n}\right)^{2}}=(1-q)\left(1-q^{3}\right)\left(1-q^{5}\right) \cdots\left(1-2 q+2 q^{4}-\cdots\right) .
$$

Throughout this paper, let $q=e^{2 \pi i \tau}$. The function $f(q)$ is convergent for $|q|<1$ and those roots of unity $q$ with odd order. For the even order roots of unity, $f(q)$ has exponential singularities. For example, $f(-0.994) \sim-1.08 \cdot 10^{31}, f(-0.996) \sim-1.02 \cdot 10^{46}, f(-0.998) \sim$ $-6.41 \cdot 10^{90}$

In order to cut out the exponential singularity at $q=-1$, Ramanujan found the function $b(q)$ which is modular form up to multiplication by $q^{-\frac{1}{24}}$, defined in his notation as

$$
b(q):=(1-q)\left(1-q^{3}\right)\left(1-q^{5}\right) \cdots\left(1-2 q+2 q^{4}-\cdots\right) .
$$

Ramanujan's last letter also inspired the problem of determining the asymptotic of the coefficients of mock theta functions such as $f(q)$. Andrews [10] and Dragonette [11] obtained asymptotic for coefficients of $f(q)$, then Bringmann and Ono [5] proved an exact formula for these coefficients. In the recent work, Folsom et al. [12,13] provided two closed formulas for the implied constant $O(1)$.

Theorem 1.1 (Folsom-Ono-Rhoades Theorem 1.1 of $[12,13])$ If $\zeta$ is a primitive even order $2 k$ root of unity, as $q$ approaches $\zeta$ radially within the unit disk, then

$$
\lim _{q \rightarrow \zeta}\left(f(q)-(-1)^{k} b(q)\right)=-4 \sum_{n=0}^{k-1}(1+\zeta)^{2}\left(1+\zeta^{2}\right)^{2} \cdots\left(1+\zeta^{n}\right)^{2} \zeta^{n+1} .
$$

\section{Remark}

(1) Theorem 1.1 makes Ramanujan's claim a special case of a more general result.

(2) Since empty products equal 1 , then Theorem 1.1 shows that

$$
\lim _{q \rightarrow-1}(f(q)+b(q))=4
$$

(3) Zudilin [14] has given an elementary proof of Theorem 1.1 by using Dyson's rank function and the Andrews-Garvan crank function in his recent work. 
In the meantime, Folsom et al. [12] proved a different form formula for the $O(1)$ constant as follows.

Theorem 1.2 (Folsom-Ono-Rhoades Theorem 1.3 of [12]) If $\zeta$ is a primitive even order $2 k$ root of unity, as $q$ approaches $\zeta$ radially within the unit disk, then

$$
\begin{aligned}
\lim _{q \rightarrow \zeta} & \left(f(q)-(-1)^{k} b(q)\right) \\
\quad & =\left\{\begin{array}{lll}
4 \sum_{n=0}^{k / 2-1}(-1)^{n} \zeta^{n+1}\left(1+\zeta^{2}\right)\left(1+\zeta^{4}\right) \cdots\left(1+\zeta^{2 n}\right), & \text { if } k \equiv 0 & (\bmod 2), \\
2+2 \sum_{n=0}^{\frac{k-1}{2}}(-1)^{n+1} \zeta^{2 n+1}(1+\zeta) \cdots\left(1+\zeta^{2 n-1}\right), & \text { if } k \equiv 1 & (\bmod 2) .
\end{array}\right.
\end{aligned}
$$

Remark The authors left an open problem as a challenge for someone to find an elementary proof to show that the constants appearing in Theorem 1.1 match those appearing in Theorem 1.2. Interesting enough, Theorem 1.2 possesses a proof in [12] that makes use of $q$-series transformations only, while Theorem 1.1 is a particular instance of a much more general result whose proof uses a machinery of mock theta functions [13]. The principal goal of this note is to give a new proof of the problem without using the relation of these two theorems.

\section{Statement of results}

As pointed out by many authors, Theorem 1.1 is a special case of a more general one, which surprisingly relates two well-known $q$-series: Dyson's rank function $R(\omega ; q)$ and the Andrews-Garvan crank function $C(\omega ; q)$. They play a prominent role in studying integer partition congruences.

In order to define these series, we let

$$
(a ; q)_{\infty}:=(1-a)(1-a q)\left(1-a q^{2}\right) \cdots,
$$

and for all $n \in \mathbf{Z}$, we denote

$$
(a ; q)_{n}:=(1-a)(1-a q) \cdots\left(1-a q^{n-1}\right) .
$$

It follows that

$$
(a ; q)_{n}=\frac{(a ; q)_{\infty}}{\left(a q^{n} ; q\right)_{\infty}}
$$

Following the paper [14] by Zudilin and the notation above, we can rewrite $f(q)$ and $b(q)$ as follows:

$$
\begin{aligned}
f(q) & :=1+\sum_{n=1}^{\infty} \frac{q^{n^{2}}}{(1+q)^{2}\left(1+q^{2}\right)^{2} \cdots\left(1+q^{n}\right)^{2}}=\sum_{n=0}^{\infty} \frac{q^{n^{2}}}{(-q ; q)_{n}^{2}}, \\
b(q) & :=(1-q)\left(1-q^{3}\right) \cdots\left(1-2 q+2 q^{4}-\cdots\right) \\
& =\left(q ; q^{2}\right)_{\infty} \sum_{n=-\infty}^{\infty}(-1)^{n} q^{n^{2}}=\frac{(q ; q)_{\infty}}{(-q ; q)_{\infty}^{2}} .
\end{aligned}
$$


For the results of Theorems 1.1 and 1.2, we have

$$
\lim _{q \rightarrow \zeta}\left(f(q)-(-1)^{k} b(q)\right)=-4 \sum_{n=0}^{k-1}(1+\zeta)^{2} \cdots\left(1+\zeta^{n}\right)^{2} \zeta^{n+1}=-4 \mu(\zeta),
$$

where

$$
\mu(q):=\sum_{n=0}^{\infty}(-q ; q)_{n}^{2} q^{n+1}
$$

and

$$
\begin{aligned}
\lim _{q \rightarrow \zeta} & \left(f(q)-(-1)^{k} b(q)\right) \\
& =\left\{\begin{array}{lll}
4 \sum_{n=0}^{\frac{k}{2}-1}(-1)^{n} \zeta^{n+1}\left(1+\zeta^{2}\right)\left(1+\zeta^{4}\right) \cdots\left(1+\zeta^{2 n}\right), & \text { if } k \equiv 0 & (\bmod 2), \\
2+2 \sum_{n=0}^{\frac{k-1}{2}}(-1)^{n+1} \zeta^{2 n+1}(1+\zeta) \cdots\left(1+\zeta^{2 n-1}\right), & \text { if } k \equiv 1 & (\bmod 2),
\end{array}\right. \\
& =\left\{\begin{array}{lll}
-4 \psi(-\zeta), & \text { if } k \equiv 0 & (\bmod 2), \\
2 \phi(-\zeta), & \text { if } k \equiv 1 & (\bmod 2),
\end{array}\right.
\end{aligned}
$$

where

$$
\psi(q):=\sum_{n=0}^{\infty}\left(-q^{2} ; q^{2}\right)_{n} q^{n+1}=\sum_{n=1}^{\infty} \frac{q^{n^{2}}}{\left(q ; q^{2}\right)_{n}},
$$

and

$$
\phi(q):=1+\sum_{n=0}^{\infty}(-1)^{n}\left(q ; q^{2}\right)_{n} q^{2 n+1}=\sum_{n=0}^{\infty} \frac{q^{n^{2}}}{\left(-q^{2} ; q^{2}\right)_{n}} .
$$

Here $\psi(q)$ and $\phi(q)$ are two third-order mock theta functions in Ramanujan's Lost Notebook.

Recall that Dyson's rank function is given by

$$
R(\omega ; q)=\sum_{n=0}^{\infty} \sum_{m \in \mathbf{Z}} N(m, n) \omega^{m} q^{n}:=1+\sum_{n=1}^{\infty} \frac{q^{n^{2}}}{(\omega q ; q)_{n}\left(\omega^{-1} q ; q\right)_{n}},
$$

where $N(m, n)$ is the number of partitions of $n$ with rank $m$.

The rank of a partition is defined to be its largest part minus the number of its parts. If $\omega \neq 1$ is a root of unity, it is known that $R(\omega ; q)$ is (up to a power of $q$ ) a mock theta function which is the holomorphic part of a harmonic Maass form of weight $1 / 2$.

The Andrews-Garvan crank function is defined by

$$
C(\omega ; q)=\sum_{n=0}^{\infty} \sum_{m \in \mathbf{Z}} M(m, n) \omega^{m} q^{n}:=\frac{(q ; q)_{\infty}}{(\omega q ; q)_{\infty}\left(\omega^{-1} q ; q\right)_{\infty}},
$$

where $M(m, n)$ is the number of partitions of $n$ with crank $m$. 
For any roots of unity $\omega, C(\omega ; q)$ is (up to a power of $q$ ) a modular form. Otherwise, we need the $q$-hypergeometric series $U(\omega ; q)$ which arises in the study of strongly unimodal sequences; it is defined by

$$
U(\omega ; q)=\sum_{n=0}^{\infty} \sum_{m \in \mathbf{Z}} \mu(m, n)(-\omega)^{m} q^{n}:=\sum_{n=0}^{\infty}(\omega q ; q)_{n}\left(\omega^{-1} q ; q\right)_{n} q^{n+1}
$$

where $\mu(m, n)$ is the number of strongly unimodal sequences of size $n$ with rank $m$.

Theorem 1.1 is a special case of the following theorem which relates these three $q$-series, here we define $\zeta_{n}:=e^{\frac{2 \pi i}{n}}$.

Theorem 2.1 (Folsom-Ono-Rhoades Theorem 1.2 of [13]) Let $1 \leq a<b$ and $1 \leq h<k$ be integers with $\operatorname{gcd}(a, b)=\operatorname{gcd}(h, k)=1$ and $b \mid k$. If $h^{\prime}$ is an integer satisfying $h h^{\prime} \equiv-1$ $(\bmod k)$, as $q$ approaches $\zeta_{k}^{h}$ radially within unity disk, then

$$
\lim _{q \rightarrow \zeta_{k}^{h}}\left(R\left(\zeta_{b}^{a} ; q\right)-\zeta_{b^{2}}^{-a^{2} h^{\prime} k} C\left(\zeta_{b}^{a} ; q\right)\right)=-\left(1-\zeta_{b}^{a}\right)\left(1-\zeta_{b}^{-a}\right) U\left(\zeta_{b}^{a} ; \zeta_{k}^{h}\right)
$$

By taking $a=1, b=2$, and $m=2 k$, so that $\zeta_{b}^{a}=-1, \zeta=\zeta_{m}^{h}$ is a primitive even order $2 k$ root of unity, Theorem 1.1 follows directly because of the fact that $f(q)=R(-1 ; q), b(q)=C(-1 ; q)$ and $\mu(q)=U(-1 ; q)$.

Based on the results above, in this note, we try to explain the relation between the two theorems, our results are as follows.

Theorem 2.2 Let $\zeta$ be a primitive even order $2 k$ root of unity, as $q$ approaches $\zeta$ radially within the unit disk, we have

(1) if $k \equiv 0(\bmod 2)$, then

$$
\lim _{q \rightarrow \zeta}\left(f(q)-(-1)^{k} b(q)\right)=-4 \mu(\zeta)=-4 \psi(-\zeta),
$$

(2) if $k \equiv 1(\bmod 2)$, then

$$
\lim _{q \rightarrow \zeta}\left(f(q)-(-1)^{k} b(q)\right)=-4 \mu(\zeta)=2 \phi(-\zeta)
$$

Obviously, we can get the following corollary from Theorem 2.2 directly.

Corollary 2.3 Let $\zeta$ be a primitive even order $2 k$ root of unity,

(1) if $k \equiv 0(\bmod 2)$, then

$$
\mu(\zeta)=\psi(-\zeta)
$$

(2) if $k \equiv 1(\bmod 2)$, then

$$
-2 \mu(\zeta)=\phi(-\zeta)
$$

\section{Remark}

(1) One can use the uniqueness property of limits to obtain the result of Theorem 2.2 by combining with the relationship between these three functions 


$$
2 \phi(-q)-f(q)=f(q)+4 \psi(-q)=b(q) .
$$

(2) In this note, we prove Theorem 2.2 by using the result related with the generating functions of convex compositions given by Andrews [15] and the online Encyclopedia of Integer Sequences [16] as well as Appell-Lerch sums, we get the desired results.

\section{Proof of the theorem}

Before we give the proof of the theorem, we would like to first introduce the recent work given by Andrews [15], which is about the theory of concave and convex compositions that linked the related generating functions to combinations of classical, false, or mock theta functions and other Appell-Lerch sums.

Following Andrews, the strictly convex composition of $n$ is defined by

$$
\sum_{i=1}^{R} a_{i}+c+\sum_{i=1}^{S} b_{i}=n,
$$

where $a_{1}<a_{2}<\cdots<a_{R}<c>b_{1}>b_{2}>\cdots>b_{S}$, and $c$ is called the central part and is $\geq 0$. We denote the number of strictly convex compositions of $n$ by $X_{d}(n)$. For example, $X_{d}(5)=6$.

The related generating functions of $X_{d}(n)$ are defined as

$$
x_{d}(q):=\sum_{n=0}^{\infty} X_{d}(n) q^{n}=\sum_{n=0}^{\infty} q^{n+1}(-q ; q)_{n}^{2} .
$$

In this work, Andrews related the above generating functions to the classical and mock theta functions as follows (Theorem 1 of [15]):

$$
x_{d}(q)=\psi(-q)+2(-q ; q)_{\infty}^{2} \alpha(-q)
$$

where $\psi(q)$ is defined in (15), and $\alpha(q)$ is defined by

$$
\alpha(q):=\sum_{n=0}^{\infty} \frac{q^{n+1}\left(-q^{2} ; q^{2}\right)_{n}}{\left(q ; q^{2}\right)_{n+1}}=\sum_{n=0}^{\infty} \frac{q^{(n+1)^{2}}\left(-q ; q^{2}\right)_{n}}{\left(q ; q^{2}\right)_{n+1}^{2}}
$$

It has been termed a second-order mock theta function by McIntosh [17].

In his deathbed letter, Ramanujan defined four third-order mock theta functions, three of them are as follows:

$$
f(q)=\sum_{n=0}^{\infty} \frac{q^{n^{2}}}{(-q ; q)_{n}^{2}}, \quad \phi(q)=\sum_{n=0}^{\infty} \frac{q^{n^{2}}}{\left(-q^{2} ; q^{2}\right)_{n}}, \quad \psi(q)=\sum_{n=1}^{\infty} \frac{q^{n^{2}}}{\left(q ; q^{2}\right)_{n}}
$$

Folsom, Ono and Rhoades used the relation between these three functions in (25) and the basic hypergeometric series

$$
F(a, b ; t)=F(a, b ; t, q):=\sum_{n=0}^{\infty} \frac{(a q ; q)_{n}}{(b q ; q)_{n}} t^{n}
$$


to prove Theorem 1.2 and to show the new forms for $\psi(q)$ and $\phi(q)$ in (15), (16), respectively.

Following Andrews, we find that

$$
x_{d}(q)=\sum_{n=0}^{\infty} q^{n+1}(-q ; q)_{n}^{2}=\mu(q)
$$

where $\mu(q)$ is defined in (13) of Theorem 1.1.

By combining with formula (28), we can deduce that

$$
\mu(q)=\psi(-q)+2(-q ; q)_{\infty}^{2} \alpha(-q)
$$

For the even $k$, let $\zeta_{k}=e^{\frac{2 \pi i}{k}}$ be any root of unity, we have the fact that pre-factor $(-q ; q)_{\infty}$ of $\alpha(-q)$ vanishes at any even root of unity, while the $\alpha(-q)$ has finite limits. As $q$ approaches an even order root of unity $\zeta_{k}$, then

$$
\lim _{q \rightarrow \zeta_{k}} \mu(q)=\lim _{q \rightarrow \zeta_{k}}\left(\psi(-q)+2(-q ; q)_{\infty}^{2} \alpha(-q)\right)
$$

namely,

$$
\lim _{q \rightarrow \zeta_{k}} \mu(q)=\mu\left(\zeta_{k}\right)=\lim _{q \rightarrow \zeta_{k}} \psi(-q)=\psi\left(-\zeta_{k}\right) .
$$

For the above fixed even $k$, we denote the primitive even order $2 k$ root of unity by $\zeta_{2 k}$. As $q$ approaches $\zeta_{2 k}$ radially within the unit disk, we have

$$
\begin{aligned}
\lim _{q \rightarrow \zeta_{2 k}}\left(f(q)-(-1)^{k} b(q)\right) & =-4 \lim _{q \rightarrow \zeta_{2 k}} \mu(q)=-4 \mu\left(\zeta_{2 k}\right) \\
& =-4 \lim _{q \rightarrow \zeta_{2 k}} \psi(-q)=-4 \psi\left(-\zeta_{2 k}\right) .
\end{aligned}
$$

The first claim in Theorem 2.2 is proved.

Folsom et al. in [12] found that a search in the Online Encyclopedia of Integer Sequences [16] shows that the values of the function $G\left(e^{-t}\right)$ have some relations with the coefficients of the expansion of $f\left(-e^{-t}\right)+b\left(-e^{-t}\right)$ while $t \rightarrow 0^{+}$, where $G(q)$ is defined by

$$
G(q):=1+\sum_{n=1}^{\infty}(-1)^{n}\left(q ; q^{2}\right)_{n}
$$

This function exists only for $q$, a root of unity. By studying the asymptotic relationship between $U\left(-1 ;-e^{-t}\right)$ and $G\left(e^{-t}\right)$, Folsom, Ono and Rhoades guessed that there might be a relation at the other roots of unity, and then they computed the first fifth odd roots of unity $\zeta_{k}$, here $\zeta_{k}=e^{\frac{2 \pi i}{k}}, k$ is odd positive integer. Surprisingly, the results of the computation clearly showed that for odd roots of unity $\zeta_{k}$,

$$
-U\left(-1 ;-\zeta_{k}\right)=G\left(\zeta_{k}\right)
$$

Combining with the Online Encyclopedia of Integer Sequences research, we can reach the conclusion that for any odd order $k$ root of unity $\zeta_{k}$, we still have the formula of (38). 
By the way, one can find that the function $G(q)$ converges in the domain $|q|<1$, because it is obvious that we can rewrite it as follows:

$$
\begin{aligned}
G(q) & :=1+\sum_{n=0}^{\infty}(-1)^{n+1}\left(q ; q^{2}\right)_{n+1} \\
& =1+\sum_{n=0}^{\infty}(-1)^{n+1}\left(q ; q^{2}\right)_{n}-\sum_{n=0}^{\infty}(-1)^{n+1} q^{2 n+1}\left(q ; q^{2}\right)_{n} \\
& =-\sum_{n=1}^{\infty}(-1)^{n}\left(q ; q^{2}\right)_{n}+\sum_{n=0}^{\infty}(-1)^{n} q^{2 n+1}\left(q ; q^{2}\right)_{n} .
\end{aligned}
$$

Applying formula (37), we then get

$$
\begin{aligned}
2 G(q) & =1+\sum_{n=0}^{\infty}(-1)^{n} q^{2 n+1}\left(q ; q^{2}\right)_{n} \\
& =1+q-q^{3}+q^{4}+q^{5}+\cdots-q^{14}-2 q^{15}+q^{16}+3 q^{17}+\cdots
\end{aligned}
$$

The search in [16] shows that this $q$-series matches the mock theta function $\phi(q)$. In fact, in the proof of Theorem 1.2, Folsom, Ono and Rhoades obtained the same form for the mock theta function $\phi(q)$ in formula (16). In this case, if $q$ is a root of unity, we have

$$
2 G(q)=\phi(q) .
$$

On the other hand, for any odd order $k$ root of unity $\zeta_{k}$, from the property of $U(\omega ; q)$, we have

$$
-U\left(-1 ; \zeta_{k}\right)=G\left(-\zeta_{k}\right)
$$

and

$$
-U(-1 ; q)=-\mu(q) .
$$

Finally, for odd integer $k$, if $\zeta_{k}$ is any primitive odd order $k$ root of unity, as $q$ approaches $\zeta_{k}$, we have

$$
\lim _{q \rightarrow \zeta_{k}}-2 \mu(q)=-2 \mu\left(\zeta_{k}\right)=\lim _{q \rightarrow \zeta_{k}}-2 U(-1 ; q)=-2 U\left(-1 ; \zeta_{k}\right)=2 G\left(-\zeta_{k}\right) .
$$

Namely,

$$
\lim _{q \rightarrow \zeta_{k}}-2 \mu(q)=-2 \mu\left(\zeta_{k}\right)=2 G\left(-\zeta_{k}\right)=\phi\left(-\zeta_{k}\right) .
$$

For the above fixed odd $k$, we denote the primitive even order $2 k$ root of unity by $\zeta_{2 k}$. As $q$ approaches $\zeta_{2 k}$ radially within the unit disk, we have

$$
\lim _{q \rightarrow \zeta_{2 k}}\left(f(q)-(-1)^{k} b(q)\right)=-4 \lim _{q \rightarrow \zeta_{2 k}} \mu(q)=-4 \mu\left(\zeta_{2 k}\right) .
$$


Since $\zeta_{2 k}^{2}=\zeta_{k}, \zeta_{2 k}$ is the root of $\zeta_{k}$. Then we get

$$
\lim _{q \rightarrow \zeta_{2 k}}(f(q)+b(q))=-4 \mu\left(\zeta_{2 k}\right)=2 \phi\left(-\zeta_{2 k}\right)
$$

We have proved the second claim in Theorem 2.2.

On the other hand, we can prove the second claim in Theorem 2.2 in a new way which is related to the properties of Appell-Lerch sums.

The definition of an Appell-Lerch sum is given by

$$
m(x, q, z):=\frac{1}{j(z ; q)} \sum_{r=-\infty}^{\infty} \frac{(-1)^{r} q^{\frac{r(r-1)}{2}} z^{r}}{1-q^{r-1} x z}
$$

where

$$
j(x ; q):=(x)_{\infty}(q / x)_{\infty}(q)_{\infty}=\sum_{n=-\infty}^{\infty}(-1)^{n} q^{\frac{n(n-1)}{2}} x^{n} .
$$

For the integers $a$ and positive integers $m$, we define

$$
J_{a, m}:=j\left(q^{a} ; q^{m}\right)
$$

and

$$
J_{m}:=J_{m, 3 m}=\prod_{i=1}^{\infty}\left(1-q^{m i}\right)
$$

Then $m(x, q, z)$ can be expressed as a bilateral sum [18]:

$$
\begin{aligned}
& \sum_{n=0}^{\infty} \frac{a^{-n-1} b^{-n}}{(-1 / a ; q)_{n+1}(-q / b ; q)_{n}} q^{n^{2}}+\sum_{n=1}^{\infty}(-a q ; q)_{n-1}(-b ; q)_{n} q^{n} \\
& \quad=\frac{(-a q)_{\infty}}{b(q)_{\infty}(-q / b)_{\infty}} j(-b ; q) m(a / b, q,-b),
\end{aligned}
$$

then we get

$$
R(\omega ; q)+(1-\omega)\left(1-\omega^{-1}\right) U(\omega ; q)=(1-\omega) \frac{j(\omega ; q)}{J_{1}} m\left(\omega^{2}, q, \omega^{-1}\right) .
$$

Let $\omega=-1$, we have

$$
R(-1 ; q)+4 U(-1 ; q)=2 \frac{j(-1 ; q)}{J_{1}} m(1, q,-1) .
$$

Recall Proposition 2.6 in [19] that

$$
\sum_{n=0}^{\infty} \frac{(-1)^{n} q^{n^{2}}\left(q ; q^{2}\right)_{n}}{\left(-x ; q^{2}\right)_{n+1}\left(-q^{2} / x ; q^{2}\right)_{n}}=m(x, q,-1)+\frac{J_{1,2}^{2}}{2 j(-x ; q)} .
$$


For the odd integer $k$ and $x=1$, we can eliminate $m(1, q,-1)$ by using the result above as follows:

$$
\begin{aligned}
R(-1 ; q)+4 U(-1 ; q) & =2 \frac{j(-1 ; q)}{J_{1}} m(1, q,-1) \\
& =\frac{j(-1 ; q)}{J_{1}} \sum_{n=0}^{\infty} \frac{(-1)^{n} q^{n^{2}}\left(q ; q^{2}\right)_{n}}{\left(-q^{2} ; q^{2}\right)_{n}}-\frac{J_{1,2}^{2}}{J_{1}} .
\end{aligned}
$$

Then we have

$$
R(-1 ; q)+\frac{J_{1,2}^{2}}{J_{1}}=-4 U(-1 ; q)+\frac{j(-1 ; q)}{J_{1}} \sum_{n=0}^{\infty} \frac{(-1)^{n} q^{n^{2}}\left(q ; q^{2}\right)_{n}}{\left(-q^{2} ; q^{2}\right)_{n}} .
$$

For odd integer $k$, if $\zeta$ is a root of unity of order $2 k$, as $q \rightarrow \zeta$, we see that the factor $\frac{j(-1 ; q)}{J_{1}}=2(-q ; q)_{\infty}^{2}$ vanishes while the summation is finite at unity, then we have

$$
\lim _{q \rightarrow \zeta}\left(R(-1 ; q)+\frac{J_{1,2}^{2}}{J_{1}}\right)=-4 U(-1 ; \zeta)
$$

where $\frac{J_{1,2}^{2}}{J_{1}}=b(q)$.

Finally, we get

$$
\lim _{q \rightarrow \zeta}(f(q)+b(q))=-4 \mu(\zeta)=2 \phi(-\zeta)
$$

The second claim in Theorem 2.2 is also completed.

\section{Competing interests}

The authors declare that they have no competing interests.

\section{Authors' contributions}

The authors declare that the study was realized in collaboration with the same responsibility. All authors read and approved the final manuscript.

\section{Author details}

'Department of Mathematics, Tongji University, No. 1239, Siping Road, Shanghai, 200092, China. ${ }^{2}$ Department of Mathematics, Weinan Normal University, Chaoyang Road, Weinan, 714000, China.

\section{Acknowledgements}

We thank the editor and the referees for their valuable suggestions to improve the quality of this article. In addition, this article is supported by the Natural Science Foundation of China under Grant (11271283), the Natural Science Foundation of Shaanxi Province of China under Grant (2012JM1021), the Foundation of CMIRI of Shaanxi Province under Grant (2013JMR11) and the Key Subject Foundation under Grant (14SXZD002).

Received: 7 May 2014 Accepted: 1 July 2014 Published: 22 Jul 2014

\section{References}

1. Andrews, GE, Berndt, BC: Ramanujan's Lost Notebook. Springer, New York (2009)

2. Zweger, S: Mock theta functions. Ph.D. Thesis (Advisor: D. Zaier), Universiteit Utrecht (2002)

3. Zweger, S: Mock $\vartheta$ functions and real analytic modular forms. In: $q$-Series with Applications to Combinatorics, Number Theory, and Physics, pp. 269-277. Contemp. Math. Am. Math. Soc., Providence (2001)

4. Bringmann, K, Ono, K: Dyson's ranks and Maass forms. Ann. Math. 171, 419-449 (2010)

5. Bringmann, K, Ono, K: The $f(q)$ mock theta function conjecture and partition ranks. Invent. Math. 165, $243-266$ (2006)

6. Zagier, D: Ramanujan's mock theta functions and their applications (after Zwegers and Ono-Bringmann). In: Séminaire Bourbaki, vol. 2007/2008, Astérisque. Exp. 326 pp. 143-164 (2009)

7. Bruinier, JH, Funke, J: On two geometric theta lifts. Duke Math. J. 125, $45-90$ (2004)

8. Berndt, BC, Rankin, RA: Ramanujan: Letters and Commentary. Am. Math. Soc., Providence (1995)

9. Griffin, M, Ono, K, Rolen, L: Ramanujan's mock theta functions. Proc. Natl. Acad. Sci. USA 110, 5765-5768 (2013) 
10. Andrews, GE: On the theorems of Watson and Dragonette for Ramanujan's mock theta functions. Am. J. Math. 88, 454-490 (1966)

11. Dragonette, L: Some asymptotic formulae for the mock theta series of Ramanujan. Trans. Am. Math. Soc. 72, 474-500 (1952)

12. Folsom, A, Ono, K, Rhoades, RC: Ramanujan's radial limits. Contemp. Math. 334, 1-12 (2012)

13. Folsom, A, Ono, K, Rhoades, RC: Mock theta functions and quantum modular forms. Forum Math., Pi 1, e2 (2013)

14. Zudilin, W: On three theorems of Folsom, Ono and Rhoades. arXiv:1309.2053v2 [math.NT] (2013)

15. Andrews, GE: Concave and convex compositions. Ramanujan J. 31, 67-82 (2013)

16. Sloane, NJA: The online Encyclopedia of Integer Sequences. http://oeis.org

17. Mclntosh, RJ: Second order mock theta function. Can. Math. Bull. 50, 284-290 (2007)

18. Ramanujan, S: The Lost Notebook and Other Unpublished Papers. Narosa Publishing House, New Delhi (1988)

19. Mortenson, ET: On the dual nature of partial theta functions and Appell-Lerch sums. Submitted. arXiv:1208.6316

10.1186/1687-1847-2014-191

Cite this article as: Chen and Zhou: Note on the problem of Ramanujan's radial limits. Advances in Difference Equations 2014, 2014:191

\section{Submit your manuscript to a SpringerOpen ${ }^{\circ}$ journal and benefit from:}

- Convenient online submission

- Rigorous peer review

- Immediate publication on acceptance

- Open access: articles freely available online

- High visibility within the field

- Retaining the copyright to your article 